\title{
Dynamic Analysis of Variable Speed Limit Control for Traffic Accident Management
}

\author{
Qingqing $\mathrm{Gu}^{1, a}$, Xingang $\mathrm{Li}^{1, \mathrm{~b}^{*}}$ and Yao Xiao, \\ School of Traffic and Transportation, Beijing Jiaotong University, Beijing 100044, China \\ 14120757@bjtu.edu.cn, lixingang@bjtu.edu.cn, xiaoy@bjtu.edu.cn \\ ${ }^{*}$ The corresponding author
}

\begin{abstract}
Keywords: Traffic accident; Variable speed limit; Cellular automata; Traffic flow
\end{abstract}
\begin{abstract}
A two-lane cellular automaton model is developed to investigate the effects of variable speed limit (VSL) control strategy on alleviating accident induced congestion and improving traffic efficiency. The impact of accident duration on traffic dynamics are analyzed by counting the downstream discharge flow rates and drawing the spatial-temporal diagram. The effectiveness of VSL control strategy is then investigated. The simulation results indicate that the longer the accident duration, the more serious the traffic congestion and the lower the system discharge flow rates are; with appropriate speed limit value and speed limit region length, traffic congestion induced by accident can be alleviated and even the system discharge flow can be increased in some extend.
\end{abstract}

\section{Introduction}

Urban expressway can provide a safe and fast driving environment and carry the main traffic demand of urban network. Traffic accidents on urban expressway might result in local congestion or even lead to a regional traffic breakdown. Thus, how to make reasonable traffic management measures to alleviate accident induced jams and improve driving safety becomes an important issue for urban traffic managers.

Variable speed limit (VSL) is widely acknowledged as a useful traffic management method in solving highway traffic congestions. The main effects of VSL perform on several aspects, such as increasing traffic flow, raising driving safety and eliminating moving jams. Jiang [1] studied traffic flow phase transition based on a car-following model. It was found that a first-order phase transition occurs under a speed limit within short region but a second-order phase transition occurs under a speed limit within long region. Lee [2] combined variable speed limits with the crash prediction mode for the evaluation of control logics. The results indicated that VSL could reduce crash potential by $5-17 \%$, by temporarily reducing speed limits during risky traffic conditions when crash potential exceeded the pre-specified threshold. Chen [3] developed VSL control based on the Kinematic Wave theory to increase discharge rates at severe freeway bottlenecks by restricting upstream demand. The results suggested that it can provide gradual speed transition at the tail of an event-induced queue and clear the queue around the bottleneck. Grumert [4] investigated a cooperative VSL system, and the results showed that VSL could harmonize traffic flow and reduce exhaust emissions. Kianfar [5] used actual deployment data to investigate the operational benefits of VSL systems. It showed that with the maximum flow prior to breakdown and the maximum flow after breakdown decreased at some locations and increased at other locations after VSL was implemented. Wang [6] used cellular automaton (CA) model-based simulations to find that there is seven, instead of the previously assumed six, states of traffic flow in the evolution process of wide moving jam (WMJ), when VSL was applied, inappropriate speed limit may induce new WMJ and exaggerates congestions in two transition ways. Wang [7] proposed an optimal coordination model of VSL based on the three-phase traffic theory to ensure the elimination of wide moving jam and to avoid the emergence of new traffic jams.

However, the study and application of VSL for urban expressway is still at an initial stage and mainly focuses on the effect of VSL at fixed congestion bottlenecks. In this paper, a two-lane 
accident model based on Kerner's three-phase traffic theory [8] is proposed to analyze the impact of accident duration on traffic dynamics, and it is found that appropriate VSL control strategy could improve system discharge flow rates.

In Sec. 2, we introduce a two-lane accident model and a VSL model and describe detailed rules considering microscopic driving characteristics and duration of traffic accident. In Sec. 3, simulations are carried out to analyze the effectiveness of VSL control strategy. Finally, conclusions are given in Sec. 4.

\section{Models}

Accident Model. According to the traffic flow characteristics of urban expressway and duration of traffic accident, a new two-lane accident model is presented. The model describes vehicles' microscopic driving behaviors.

When a traffic accident happened on the urban expressway, vehicles would react to the sudden incident. Generally, vehicles converge to a region, then change into non-accident lane upstream the accident sites, and finally sequentially leave bottleneck. Therefore, urban expressway is divided into basic sections and accident sections. The latter includes convergence region and lane-changes region.

Two-lane urban expressway consists of Lane1 and Lane2. Lane 1 is the accident lane and Lane2 is the non-accident lane. As illustrated in Fig. 1, the marks of c, d, e, f represent the boundary of a certain region, respectively. Where e-f is accident region, $d-e$ is lane-changes region and c-d stands for convergence region. The vehicle which is nearest to the convergence region upstream in a specific lane is called lead car.

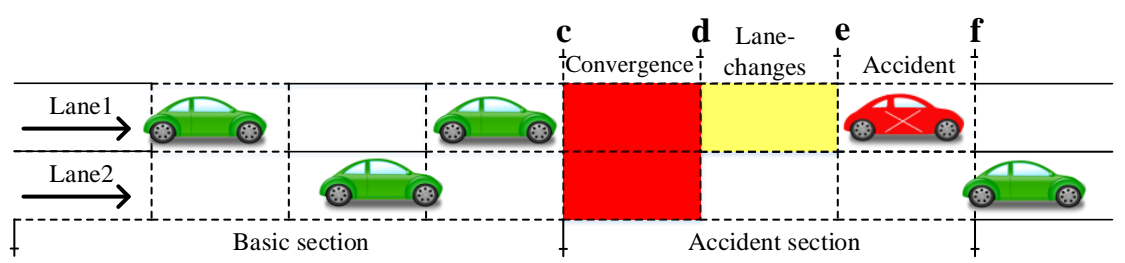

Figure 1. The feature of two-lane influenced by accident

(1) Lane-changes rules in basic section

Other vehicles in basic section except the lead cars follow symmetry lane-changes rules. The rules are introduced as follows, change motivation and safety conditions:

$$
d_{n}^{+}<\min \left(v_{n}+1, v_{\max }\right), \quad d_{n, \text { other }}^{+}>d_{n}^{+} \quad \text { and } \quad d_{n, \text { other }}^{-}>d_{\text {safe }}
$$

$\mathrm{d}_{\mathrm{n}}^{+}$is the space gap between the vehicle and preceding vehicle in this lane;

$\mathrm{d}_{\mathrm{n}, \mathrm{other}}^{+}$is the space gap between the vehicle and preceding vehicle in other lane;

$\mathrm{d}_{\mathrm{n}, \text { other }}^{-}$is the space gap between the vehicle and trailing vehicle in other lane;

(2) Update rules in basic section

Kerner-Klenov-Wolf (KKW) model is adopted here to simulate the forward motion of vehicles. The parallel updating rules are as follows:

Certain updating rules:

$$
\begin{aligned}
& \tilde{\mathrm{v}}_{\mathrm{n}}\left(\mathrm{t}_{1}\right)=\max \left\{0, \min \left\{\mathrm{v}_{\text {max }}, \mathrm{v}_{\text {safe }}(\mathrm{t}), \mathrm{v}_{\text {des }}(\mathrm{t})\right\}\right\} \\
& \mathrm{v}_{\text {des }}(\mathrm{t})=\left\{\begin{array}{c}
\mathrm{v}_{\mathrm{n}}(\mathrm{t})+\mathrm{a} \tau \quad: \text { for } \mathrm{d}_{\mathrm{n}}^{+}>\mathrm{D}\left[\mathrm{v}_{\mathrm{n}}(\mathrm{t})\right]-\mathrm{l} \\
\mathrm{v}_{\mathrm{n}}(\mathrm{t})+\mathrm{a} \tau: \text { for } \mathrm{d}_{\mathrm{n}}^{+} \leq \mathrm{D}\left[\mathrm{v}_{\mathrm{n}}(\mathrm{t})\right]-\mathrm{l} \text { and } \mathrm{g}_{\mathrm{n}} \geq 0 \\
\mathrm{v}_{\mathrm{n}}(\mathrm{t})-\mathrm{b} \tau: \text { for } \mathrm{d}_{\mathrm{n}}^{+} \leq \mathrm{D}\left[\mathrm{v}_{\mathrm{n}}(\mathrm{t})\right]-\mathrm{l} \text { and } \mathrm{g}_{\mathrm{n}}<0
\end{array}\right.
\end{aligned}
$$

Stochastic updating rules: 


$$
\begin{aligned}
& \mathrm{v}_{\mathrm{n}}(\mathrm{t}+1)=\max \left\{0, \min \left\{\tilde{\mathrm{v}}_{\mathrm{n}}\left(\mathrm{t}_{1}\right)+\tau \eta_{\mathrm{n}}, \mathrm{v}_{\mathrm{n}}\left(\mathrm{t}_{1}\right)+\mathrm{a} \tau, \mathrm{v}_{\text {free }}, \mathrm{v}_{\text {safe }}\right\}\right\} \\
& \mathrm{x}_{\mathrm{n}}(\mathrm{t}+1)=\mathrm{x}_{\mathrm{n}}(\mathrm{t})+\mathrm{v}_{\mathrm{n}}(\mathrm{t}+1) \tau
\end{aligned}
$$

Parameters:

$$
\begin{gathered}
D\left(v_{n}(t)\right)=l+k v_{n}(t) \tau \\
\eta_{n}=\left\{\begin{array}{cc}
-b & g_{n}=v_{n+1}(t)-v_{n}(t) \\
a & : \text { if } p_{b} \leq r<p_{b}+p_{a} \\
0 & \text { : otherwise }
\end{array}\right. \\
p_{b}\left(v_{n}\right)=\left\{\begin{array}{lll}
p_{0}: & \text { if } v_{n}=0 \\
p & : & \text { if } v_{n}>0
\end{array} \quad p_{a}\left(v_{n}\right)= \begin{cases}p_{a 1}: & \text { if } v_{n}<v_{p} \\
p_{a 2}: & \text { if } v_{n} \geq v_{P}\end{cases} \right.
\end{gathered}
$$

In Eqs. $1-8, \mathrm{n}=0,1,2 \ldots$ is the number of vehicles. $\tau$ is discrete time steps. $\mathrm{t}$ is current time, $l$ is the length of a vehicle. $v_{\max }$ marks the maximum speed. $\tilde{v}_{n}(t)$ is the speed of vehicle $\mathrm{n}$ at the time $t$ without random fluctuation. $v_{n+1}(t)$ is the speed of the preceding vehicle. $g_{n}$ represents the difference of the vehicle and the preceding one. $v_{\text {safe }}(t)$ and $v_{\text {free }}$ are safety speed and free speed respectively. $x_{n}(t), x_{n}(t+1)$ are the location at current and next step. $D\left(v_{n}(t)\right)$ is a synchronization space gap. $\eta_{n}$ is the random fluctuation of speed; a and b respectively represent acceleration and deceleration of vehicles. $p_{a}\left(v_{n}\right)$ and $p_{b}\left(v_{n}\right)$ are the probability of a and $\mathrm{b}$. $\mathrm{r}$ is a random number which is uniformly distributed between 0 and $1 . k, v_{p}, p_{a 1}, p_{a 2}, p, p_{0}$ are constants.

(3) Lane-changes rules in accident section

In the accident section, lane-changes can only happen in lane-changes region d-e. The lane-changes rules are relaxed [9] as follows.

$$
d_{n, \text { other }}>0 \text { and } \mathrm{d}_{\mathrm{n}, \text { back }}>\mathrm{d}_{\text {safe }}
$$

(4) Update rules in accident section

As shown in Fig. 2, special update rules in accident section are adopted in order to achieve zigzag effect when vehicles pass through the bottleneck. Assume that the lead cars on the two lanes are Leadcar1 and Leadcar2.

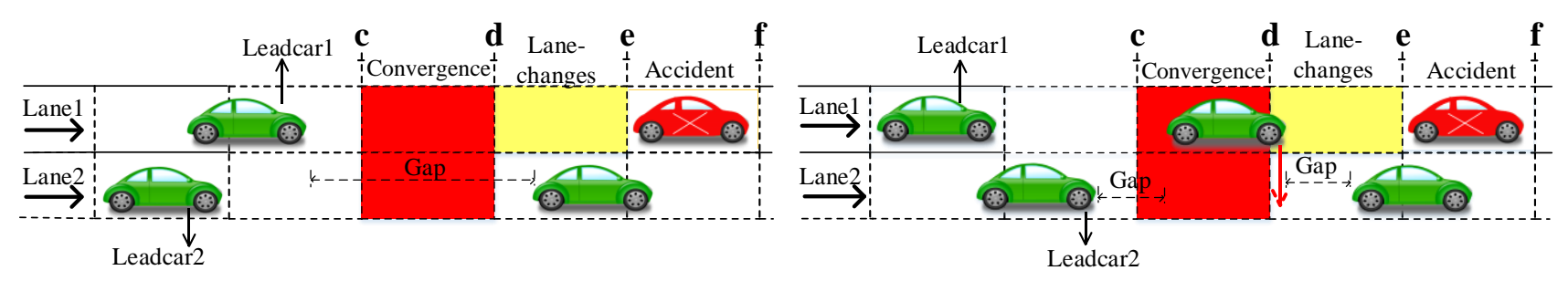

Figure 2. Update rule (1) and update rule (2)

At the current time step, when there is no vehicle in the convergence region, if Leadcar1 is in front of or has the same position with Leadcar2, Leadcar1 will have a priority to enter convergence region. Otherwise, Leadcar2 will have the priority. Also the lead car will update their velocity and position according to the preceding vehicle on the non-accident lane.

At the current time step, when there is one vehicle in the convergence region, then the lead car on the other lane has priority to enter convergence region, and it will update velocity and position according to the preceding vehicle in the convergence region. The rules can ensure the car in convergence region have enough space to do converging and lane-changes.

Variable Speed Limit (VSL) Model. When traffic accident occurs on urban expressway, congestion at accident bottleneck will rapidly spread upstream. VSL control strategy can alleviate traffic congestion and avoid the emergence of new jams. The speed limit region is located at 
upstream bottleneck (see Fig. 3). In order to achieve smoothly converging and passing, collaborative updating is used in section b-c. Vehicles at this section will update velocity and position not only according to the preceding vehicle at current lane, but also considering the other lane. Thus the gap is calculated by $\operatorname{Gap}=\operatorname{Min}\left(d_{n}^{+}, d_{n, \text { other }}^{+}\right)$. When the two vehicles on different lanes have the same position, the vehicle on lane 1 has priority, so the vehicle on lane 1 will be the preceding vehicle.

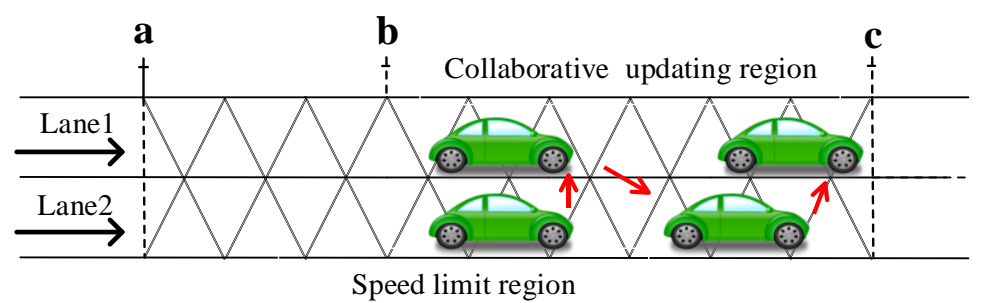

Figure 3. VSL model and collaborative updating rules

Boundary Conditions. In this model, open boundary conditions are adopted. Vehicles entering zone is located at $0 \sim v_{\max }$ cells. At each time step, the position of last vehicle $x_{\text {last }}$ is detected. If $x_{\text {last }}>v_{\max }$ means there is no vehicle in the entering zone, then with probability $P_{\text {enter }}$ add new car at the location $\min \left(x_{\text {last }}-v_{\max }, v_{\max }\right)$, with velocity of $v_{\max }$.

When updating is completed at each time step, detect the position of lead vehicle. If the position beyond the length of road $x_{\text {lead }}>L$, the lead vehicle should be removed.

\section{Simulation and Analysis}

Numerical Simulation and Result Analysis of the Accident Model. In this model, the length of road is 12000 cells and each cell corresponds to 0.5 meters. Traffic accident is located at 10000th cell. Each time step represents 1s, and the total simulation time steps is $1200 \mathrm{~s}$. Each vehicle has a length of 15 cells $(7.5 \mathrm{~m})$. The maximum speed $v_{\max }$ is 60 cells $/ \mathrm{s}$, which equals to $108 \mathrm{~km} / \mathrm{h}$. In each time step, the vehicle will adjust acceleration or deceleration according to traffic environment, respectively marked as a and $\mathrm{b}, \mathrm{a}=8$ cells $/ \mathrm{s}^{2}\left(4 \mathrm{~m} / \mathrm{s}^{2}\right)$ and $\mathrm{b}=5$ cells $/ \mathrm{s}^{2}\left(2.5 \mathrm{~m} / \mathrm{s}^{2}\right)$.

Accident duration (D) is different due to accident severity, occurrence time and treatment measures. According to the division of HCM 2000, the total influence time of accident is divided into detection time, response time, clearance time and recovery time. However we mainly study the first three stages, and the accident duration is the time from the accident occurrence to clearance. In this paper, the variables $\mathrm{D}$ are assigned to $10 \mathrm{~min}, 15 \mathrm{~min}$, and $20 \mathrm{~min}$. Other parameters are constants $k=2.55, p_{0}=0.2, v_{p}=28$. In order to eliminate the influence of unstable data, the simulation results give up the statistical data of first 1000 time steps.

(1) The impact of accident duration on traffic flow parameters

During the simulation, system discharge flow rates, average velocity are counted and spatial-temporal diagram under different accident duration are plotted. In this paper, system discharge flow rates is defined as the stable saturation flow which can pass through the bottleneck of accident in unit time, which can be obtained by counting the number of vehicles driving out of the system at the end of road. Critical entry rates is defined as the entry rates when system discharge flow rates reaches a stable and saturated state.

Firstly, we analyze the relationship between system discharge flow rates and entry rates, as shown in Fig. 4. With the increasing of entry rates, system discharge flow rates increases linearly and then goes into the saturated state after a small drop when entry rates is greater than critical entry rates. Meanwhile, traffic breakdown occurs with phase transition from free flow to congested flow. By simulation, we can obtain that the saturated discharge flow rates of two-lane system is $1.2203 \mathrm{veh} / \mathrm{s} / \mathrm{lane}$, namely $2196 \mathrm{veh} / \mathrm{h} / \mathrm{lane}$ under no accident condition, the value is very close to the design capacity of $2200 \mathrm{veh} / \mathrm{h} / \mathrm{lane}$ of urban expressway specification, which can confirm that the 
accident model can greatly simulate the actual traffic conditions. When accident duration are 10min, $15 \mathrm{~min}$, 30min, the saturated discharge flow is approximated to $0.6418 \mathrm{veh} / \mathrm{s}, 0.5206 \mathrm{veh} / \mathrm{s}$, $0.4103 \mathrm{veh} / \mathrm{s}$ respectively, which is dropped down to $52.59 \%, 42.66 \%, 33.62 \%$ compared with no accident case. At the moment, corresponding critical entry rates are respectively $0.26,0.32,0.36$. By comparing all situations, we can conclude that system discharge flow rates of two-lane accident model presents rising linearly to saturation state and the difficulty of reaching saturation flow rates is a decreasing relationship with accident duration, exactly the longer duration, the lower system discharge flow rates and the easier to reach saturation state.

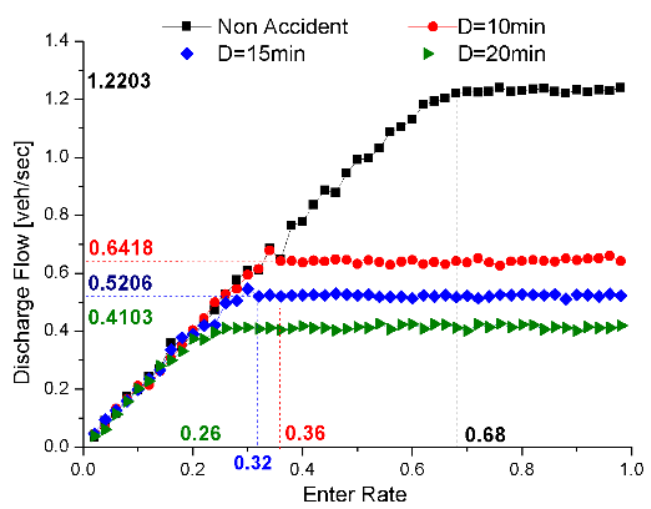

Figure 4. The relationship between system discharge flow rates and entry rates

In general, when traffic accident occurs, the accident lane will often produce more serious congestion. Therefore, in order to evaluate the validity of two-lane accident model, we do analysis respectively from flow level and velocity level. We select $\mathrm{D}=20 \mathrm{~min}$ as one example, average velocity is the average value of all vehicles in certain region (consistent with the length of the VSL region in the following context, for 2000 cells) at upstream the accident sites. It is mainly due to the region is closing to accident sites, where vehicles can reflect the more precise micro behaviors. As shown in Fig. 5 and Fig. 6, average velocity will decrease sharply when entry rates is not small and become constant as enter rates further increases. We can clearly get a situation, traffic flow states in lanes is consistent despite accident. That is to say that even if the accident happened in the lane1, but there is not serious breakdown or queue and which is similar to the traffic flow moving state of non-accident lane. Above all, it shows that the rules that vehicles pass through accident bottleneck alternately, can make full use of the space of two lanes and offer the efficiency to pass through the bottleneck.

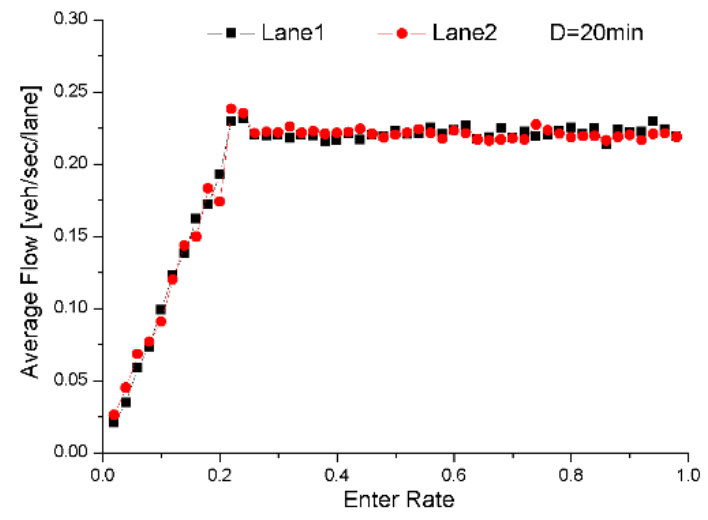

Figure 5. System discharge flow rates of each lane

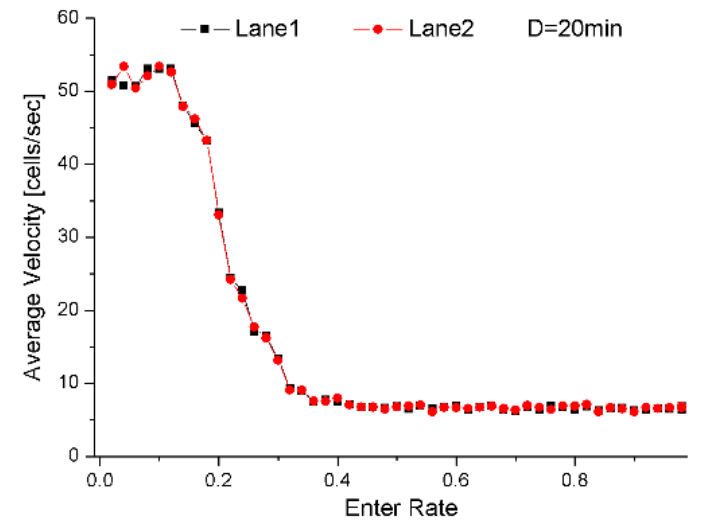

Figure 6. Average velocity of each lane

(2) The impact of accident duration on congestion waves

It is mentioned above that different accident duration have different critical entry rates. In order to study the dynamic evolution of traffic flow in accident section in detail, drawing respectively spatial-temporal character of free flow and congestion flow. As shown in Fig. 7, it is 
spatial-temporal diagram with current system entry rates is lower than critical entry rates. For $\mathrm{D}=$ $10 \mathrm{~min}$, entry rates of a and $\mathrm{d}$ is 0.3 (converted into system discharge flow rates is $1080 \mathrm{veh} / \mathrm{h} / \mathrm{lane}$ ). We can conclude that all vehicles on the road are in free flow state and traffic density is small, average velocity is relatively higher. Because current system entry rates is lower than critical entry rates, that is $0.3<0.32$, a relatively small range of congestion zone with low average velocity in accident site will be formed, meanwhile the congestion is alternately appeared or dissipated. Similar to above, the entry rates of $\mathrm{b}$ and $\mathrm{e}$ is 0.23 , and $\mathrm{c}$ or $\mathrm{f}$ is 0.2 , which is much lower than their critical entry rates: $0.25,0.21$.

As Fig. 8 shown is that the spatial-temporal diagram when current system entry rates is higher than critical entry rates. Also when $\mathrm{D}=10 \mathrm{~min}$, the entry rates of $\mathrm{a}$, e is 0.3 (converted into system discharge flow rates is $1440 \mathrm{veh} / \mathrm{h} / \mathrm{lane}$ ), which is higher than system discharge flow rates. There will form a wide range of congestion zone with lots of vehicles, and the propagation speed of congestion waves can quickly influence upstream. We can clearly see that it is a sharp contrast between upstream and downstream. For the former, traffic density is larger and the vehicles are stop-and-go. However, for the latter, changing motivation of vehicles in downstream is much poor that there is only few sparse traffic trajectory on the accident road. Similar to above, the entry rates of b, e, c, f all is 0.4 , which is larger than critical entry rates. In this paper, we assume that with the end of accident, horizontal length of bottlenecks upstream congestion zone reflects the propagation ability of congestion waves. The red horizontal line, is plotted in Fig. 8, showed that with the same entry rates different propagation ability of congestion under different accident duration time. By comparing, we can find that the propagation ability of congestion waves is in direct proportion to accident duration time, which is mainly because that with the increasing of accident duration, the more vehicles located in upstream will be forced to change the movement state. It will cause a kind of propagative chain reaction and exacerbate congestion zone. Without any traffic management measures, the influence of accident before removed will take long time to recover.

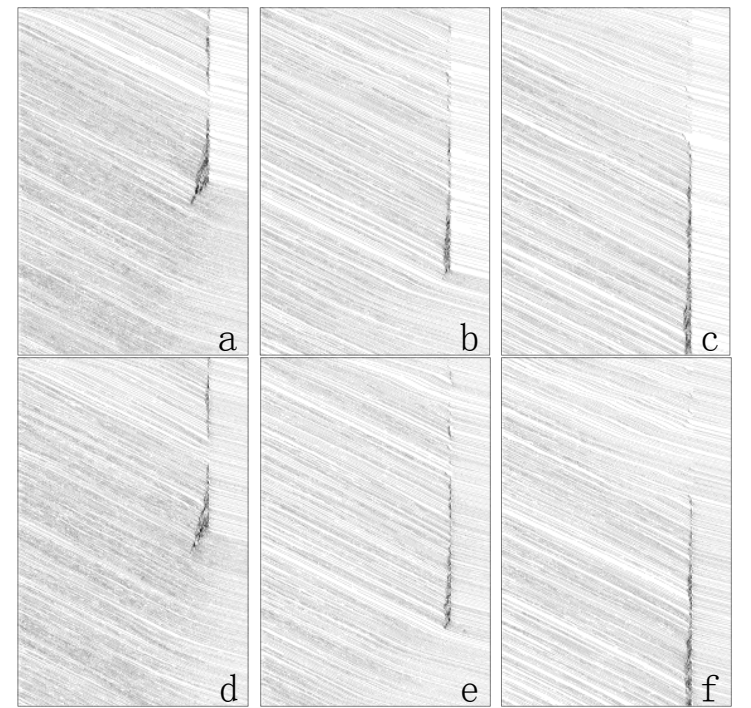

Figure 7. Spatial-temporal diagram of each lane in the case of different accident duration when current entry rates is lower than critical entry rates $\mathrm{a} / \mathrm{b}, \mathrm{b} / \mathrm{e}, \mathrm{c} / \mathrm{f}$ respectively represent $D$ equals to $10 \mathrm{~min}, 15 \mathrm{~min}, 20 \mathrm{~min} ; \mathrm{a} / \mathrm{b} / \mathrm{c}, \mathrm{d} / \mathrm{e} / \mathrm{f}$ respectively illustrates lane 1 and lane 2
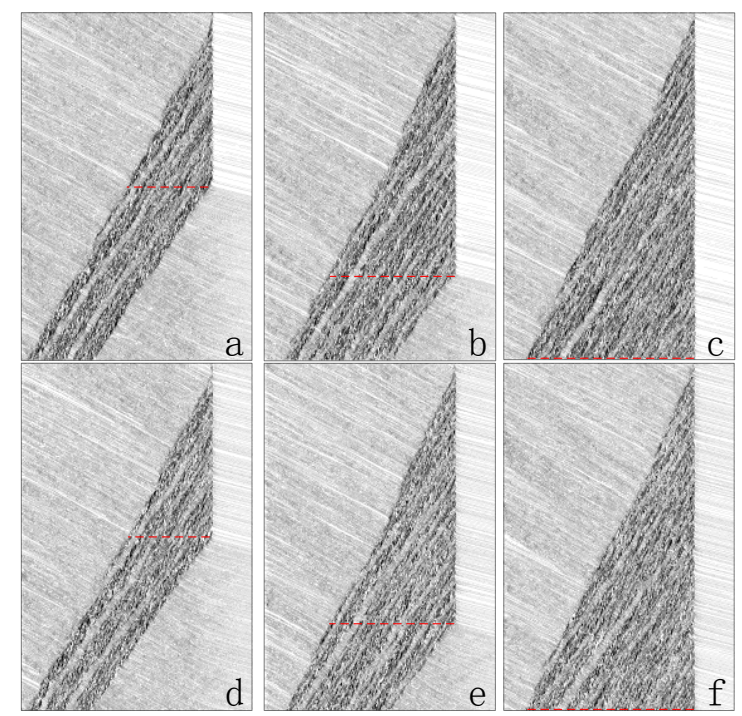

Figure 8. Spatial-temporal diagram of each lane in the case of different accident duration when current entry rates is higher than critical entry rates $\mathrm{a} / \mathrm{b}, \mathrm{b} / \mathrm{e}, \mathrm{c} / \mathrm{f}$ respectively represent $\mathrm{D}$ equals to $10 \mathrm{~min}, 15 \mathrm{~min}, 20 \mathrm{~min} ; \mathrm{a} / \mathrm{b} / \mathrm{c}, \mathrm{d} / \mathrm{e} / \mathrm{f}$ respectively illustrates lane 1 and lane 2

Numerical Simulation and Results Analysis of VSL Model. In VSL model, we assumed that the speed limit region ranges from 500th cell to 10000th cell, the speed limit changes from 10cells/s to 50cells/s, and the length of collaborative updating section is 200cells. This article respectively analyzes the influence of system discharge flow rates in two-lane under various conditions, and also evaluate the effect of VSL strategy in the evolution of traffic flow. 
(1) The impact on traffic flow parameters by VSL strategy

Firstly, the length of speed limit region is set as 2000cells and five speed limit values, 10cells/s, 20cells/s, 30cells/s, 40cells/s, 50cells/s are selected. When $\mathrm{D}=20 \mathrm{~min}$, system discharge flow rates are $0.2573,0.3913,0.4357,0.4285$ and 0.4105 respectively (see Fig. 9). One can see that the system discharge flow rates is maximum when the speed limit is 30cells/s. This indicates that if speed limit value is too small, the upstream traffic flow will be greatly suppressed and then the road facilities cannot be fully used. However, if speed limit value is too large, it will cut the effect of upstream VSL control strategy due to flow load in upstream and the efficiency of bottleneck will be reduced because of server queues. Moreover, the longer the duration of accident, the system discharge flow rates are affected by the VSL more obviously.

And then, the impact of the speed limit region length is studied. Now the speed limit value is set as 30cells/s and the length of speed limit region are set as 400cells, 1000cells, 2000cells, 5000cells and 10000cells respectively. It is obvious that the system discharge flow rates is not affected by the length of speed limit region. The speed limit region is just an artificial bottleneck. The bottleneck can be compared to conduit, speed limit value is the diameter of the pipe and the length of limit region is the length of pipe. Therefore, with the same speed limit value, system discharge flow rates has little difference whether the limit region is long or short. However, short speed limit region will not conducive to make sufficient reaction time to adjust VSL control and pose a higher security risks. Therefore setting up appropriate values of the speed limit and the length of speed limit region, traffic congestion could be alleviated.
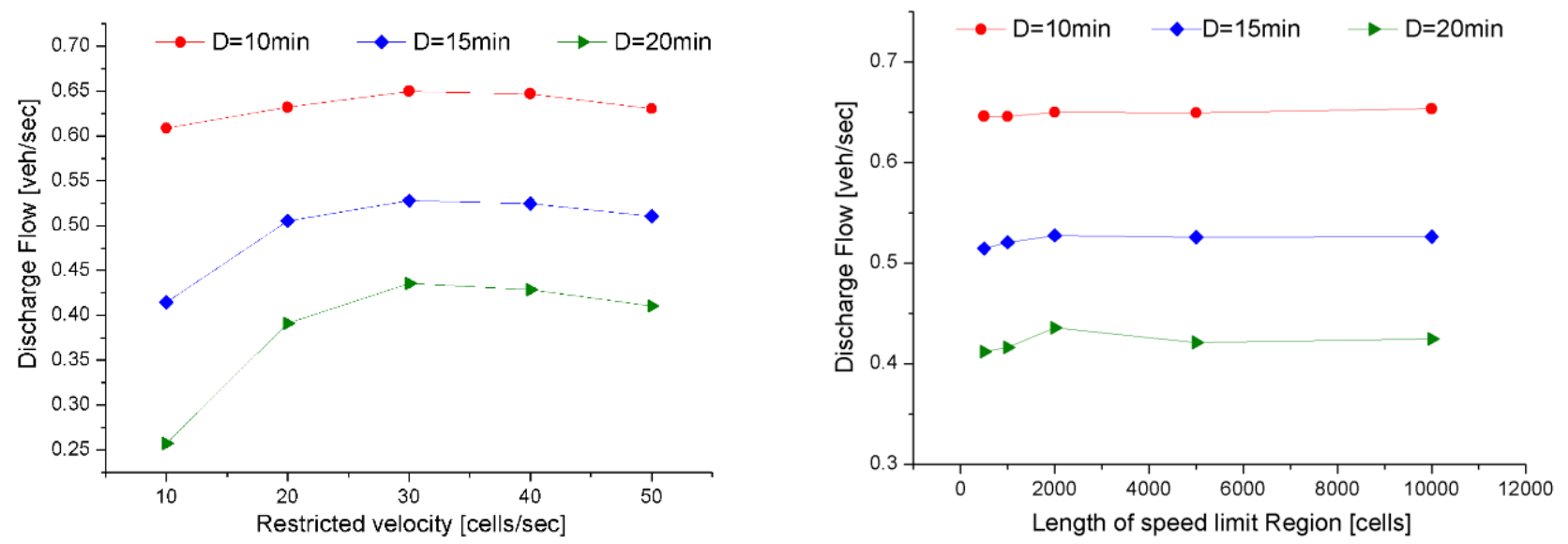

Figure 9. System discharge flow rates under different restricted velocity and different limit region

Fig. 10 presents the Spatial-temporal diagram with and without VSL control strategy. The speed limit region has the length of 2000cells and the speed limit value is 30cell/s. One can see that the vehicles which are in the speed limit region will slow down due to VSL control. Therefore, the formation of traffic congestion is delayed and the length of traffic jam region is reduced [10]. In addition, there are significant differences in the traffic flow state of collaborative updating section and other region, traffic density of this section is much lower than others, meanwhile the average velocity is much larger than other parts and become an area looks like isolation zone. It not only segregate the congested traffic but also let the upstream traffic grooming into a smooth and orderly state. And thus the system discharge flow rates is slightly raised and safety driving condition is greatly improved. 

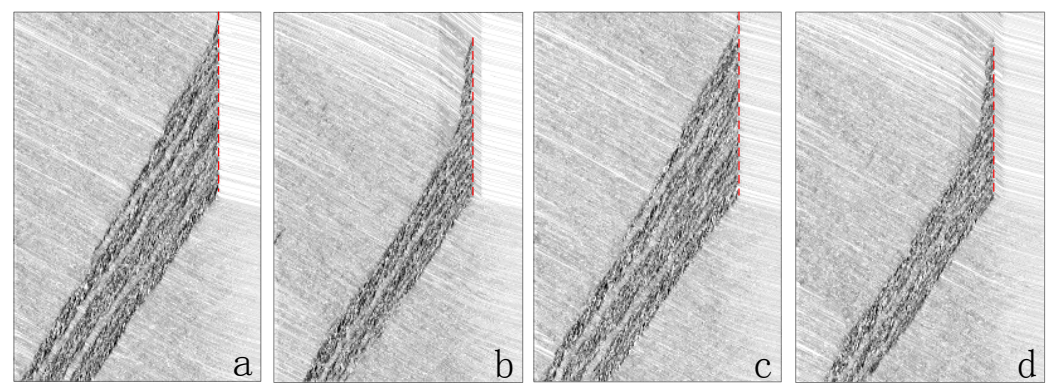

Figure 10. Spatial-temporal diagram with and without VSL control strategy (a/c, b/d respectively represent non- strategy and VSL strategy; a/b, c/d respectively illustrates lane 1 and lane 2.)

\section{Conclusion}

In this paper, we studied traffic flow dynamics under traffic accident and VSL control conditions. The main conclusions include, 1) Accident model with microscopic convergence and lane-changes behaviors can reproduce the characteristics of urban expressway traffic flow; 2) A longer accident duration leads to a smaller system discharge flow rates; 3) An appropriate value of speed limit can smooth traffic states and raise system discharge flow more or less. In this way, traffic congestion induced by accident could be alleviated in some extend.

In this paper, the speed limit region only contains one segment. In our future work, speed limit region with more than one segment, which has different speed limit values, will be investigated. Then the speed limit values among different segments should be coordinated. Thus optimization models are needed to solve this problem. Furthermore, the proposed VSL control strategy will be verified by empirical traffic data.

\section{Acknowledgements}

This work is financially supported by grants from the National Natural Science Foundation of China (Nos. 71371001, 71210001, and 71471012).

\section{References}

[1] R. Jiang and Q.S. Wu: Physica A: Statistical Mechanics and its Applications, Vol. 322 (2003) p. 676.

[2] C. Lee, B. Hellinga and F. Saccomanno: Transportation Research Part C: Emerging Technologies, Vol. 14 (2006) No.3, p. 213.

[3] D. Chen and S. Ahn: Transportation Research Part C: Emerging Technologies, Vol. 51 (2015) p. 210.

[4] E. Grumert, X. Ma and A. Tapani: Transportation research part C: Emerging Technologies, Vol. 52 (2015) p.173.

[5] J Kianfar, P Edara and C Sun: Journal of Intelligent Transportation Systems, Vol. 19 (2006) No.4, p.385.

[6] Y. Wang, Y.I. Zhang and J. Hu: International Journal of Modern Physics C, Vol. 23 (2012) No.9, p.1250060.

[7] Y. Wang, J. Hu and L. Li et al: International Journal of Modern Physics C, Vol. 25 (2014) No.9, p. 1450038 .

[8] B.S. Kerner, S.L. Klenov and D E Wolf: Journal of Physics A: Mathematical and General, Vol. 35 (2002) No.47, p.9971.

[9] B.S.Kerner, S.L. Klenov, M. Schreckenberg: Physical Review E, Vol. 84 (2011) No.4, p. 046110. 
[10]Y. Wang, Y.I. Zhang and J. Hu: International Journal of Modern Physics C, Vol. 23 (2012) No.9, p. 1250060. 